\title{
Comparison of resource distribution metrics on multi-resource projects
}

\author{
Ö.H. Bettemir ${ }^{*}$ and T. Erzurum \\ İn̈nü University, Department of Civil Engineering, Malatya, Turkey
}

\begin{abstract}
Resource leveling is implemented to reduce the fluctuations of the daily resource usage and the peak resource usage throughout the construction period. Measurement of peak resource demand is straightforward, while fluctuations of resource distribution are difficult to evaluate. Therefore, many resource distribution metrics are proposed in order to measure the fluctuations of the resource distribution. In this study, minimum moment, absolute difference, sum of the squares, and minimum deviation resource distribution metrics are compared by implementing resource distribution on four case study problems obtained from the previous studies. The case study problems are optimized by exhaustive enumeration in order to ensure that the global optimum is obtained for each resource distribution metric. Recently the comparison was conducted for small projects. In this study, larger projects are evaluated to examine the effects of the project size and the search domain. Optimized resource distribution metrics are compared in terms of peak resource demand and the overall resource distribution histogram. Similarity to the standard 'S' curve is expected for good fit resource distribution. The metrics are also compared in terms of easiness of implementation and computational demand. Results of the analyses revealed that minimum moment metric provided lower peak resource demand among the other resource distribution metrics. In addition to this, computational demand and easiness of implementation of the minimum moment metric is the most suitable. The results of this research can be beneficial for the project managers through the selection of appropriate resource distribution metric.
\end{abstract}

\section{Keywords}

Resource leveling problem; Resource distribution metrics; Exhaustive enumeration; Optimization

Received: 29 May 2019; Accepted: 27 June 2019

ISSN: 2630-5771 (online) @ 2018 Golden Light Publishing All rights reserved.

\section{Introduction}

Early start and finish time of activities are determined by critical path method. Daily resource usage throughout the construction period can be computed by considering the resource demand of the activities and the time of the execution of the corresponding activities. Initial resource usage chart illustrates the daily resource demand with all of the activities are started at early start time. However, the initial daily resource diagram may not be the most suitable one. This is because a more suitable resource distribution may be achieved by delaying uncritical activities. If maximum resource demand throughout the construction period and number of idle workers can be reduced, more suitable resource distribution will be obtained. If the project duration is kept constant, in other words resource usage histogram is leveled without causing a delay in construction duration; the endeavor is called resource leveling.

Resource leveling has significant benefits for the contractors. Some of the benefits can be listed as it reduces the amount of idle workers and construction machines and reduces the construction

* Corresponding author

Email: onder.bettemir@inonu.edu.tr 
cost. In addition to this, it reduces the amount of frequent release and re-hire of construction workers. This is also important since a worker may find another job when he is idle and experienced and accustomed workers to the habits of the constructions site might be lost. In addition to this if the executed job is an international project, release and re-hire option may not be applicable for most of the workers. Resource leveling may reduce the peak resource demand which can lead to reduction in the overhead costs since smaller accommodation and job site utility structures would be needed. Because of the mentioned benefits, solution of resource leveling problem is important.

Resource leveling problem is determined as non-deterministic polynomial-time hard (NP-Hard) type optimization problem [1-2]. Therefore, optimum solution of Resource Leveling Problem (RLP) becomes very difficult for large projects. Therefore many researchers studied on the solution of resource leveling problem. Linear-programming based resource-leveling algorithm is developed by Karaa and Nasr [3]. Easa [4] proposed integerlinear optimization model to solve this problem. Demeulemeester and Herroelen [5] developed branch-and-bound algorithm. Non-serial dynamic programming model is proposed by Bandelloni et al. [6]. Neumann and Zimmermann [7] introduced branch-and-bound and truncated branch-and-bound procedures. Mutlu [8] developed a branch-andbound algorithm for the optimum solution of small and medium size projects. In addition to mathematical methods evaluation of whole search domain of RLP is executed by Erzurum [9]. Whole search domain is scanned by delaying uncritical activities systematically to evaluate every feasible solution alternative.

Besides the optimum solution of resource leveling problem evaluation of fluctuations of resource distribution histogram, detection of amount of idle workers, and suitability of the resource distribution for the contractors are important criteria. The implemented resource distribution metric should penalize unsuitable resource distribution properly based on the aforementioned demands. Since no resource distribution metric can fit to all of the optimization criteria some of the requests are fulfilled. Each resource distribution metric has different objective functions. Therefore implemented resource distribution metric provides a different resource distribution histogram. The decision maker should know the characteristics of the resource distribution metrics before the optimization process.

In this study; minimum moment, absolute difference, sum of the squares, and minimum deviation metrics are examined on the four construction projects obtained from the literature. Resource leveling projects are derived and are solved by using the aforementioned resource distribution metrics. The results are compared by considering the peak resource demand, and fluctuations throughout the construction period. Findings of this study can be beneficial for the project managers for the selection of proper resource distribution metric.

\section{Theory}

Many resource distribution metrics have been developed because one metric cannot fit to the whole resource leveling endeavor. Burgess and Killebrew proposed minimizing the sum of the squares of the daily resource demand [10]. The formula of minimum moment metric is given in Eq.

$$
M M=\sum_{i=1}^{S} r_{i}^{2}
$$

where ri is the daily resource demand in the ith day and $S$ is the duration of the project. Daily resource demand shows the number of workers for a particular workday.

Easa [4] developed a resource leveling metric which considers the sum of the Absolute Difference (AD) of the resource demands of consecutive days. Equation of $\mathrm{AD}$ metric is given in Equation (2).

$$
A D=\sum_{i=2}^{S}\left|r_{i}-r_{i-1}\right|
$$

Easa [4] also proposed minimizing the sum of the absolute value of the deviation of the resource 
usage from the mean of the daily resource demand. Equation (3) illustrates the minimum deviation (MD) metric.

$$
M D=\sum_{i=1}^{S}\left|r_{i}-\bar{r}\right|
$$

where is the mean of the resource demand or a predefined value. Ahuja [11] developed a resource leveling metric which minimizes the sum of the square of the differences of daily resource usages between the consecutive days. Square of difference (SD) metric is given in Eq. (4).

$$
S D=\sum_{i=2}^{S}\left(r_{i}-r_{i-1}\right)^{2}
$$

In the literature many resource distribution metrics are proposed by the researchers. Some of the metrics aimed to reduce the amount of idle workers by penalizing the fall in the resource distribution followed by a rise. This resource distribution metric is called Release and Re-Hire (RRH) metric which is developed by El-Rayes and Jun [12]. Also a similar metric Resource Idle Day (RID) metric is proposed by the same researchers. In order to keep the volume of this study reasonable, these two resource leveling metrics are not included.

Solution of the case study problems are performed by exhaustive enumeration. This technique scans the whole search domain by trying all of the feasible solutions which can be obtained by delaying the uncritical activities. Determination of the whole search domain of the RLP is performed by Erzurum and Bettemir [13] where small and medium sized projects are examined and their search domains are determined. The evaluation of the whole search domain is executed by Erzurum and Bettemir [14], Erzurum [9], and Bettemir and Erzurum [15]. Computations are performed by a macro written on a spreadsheet application.

\section{Case study problems}

In this section four case study problems are used for the comparison of resource distribution metrics. The case study problems are derived from literature in which the problem size is suitable for the exhaustive enumeration. All of the case study problems are solved for the four resource distribution metrics to reveal the performances of them. The solution algorithm is executed on spreadsheet program. The problems are solved on 3 Ghz Pentium IV CPU with 2 GB RAM.

The case study problems are solved by the researchers in order to test the optimization algorithm which they had developed. In this study, performances the resource distribution metrics are compared. The solution is obtained by exhaustive evaluation therefore all of the solutions are exact. The selected problems are medium sized problems so that optimum solution is guaranteed in reasonable computation time. In addition to this, project durations are also reasonable that resource distribution diagrams can be plotted in the manuscript without occupying too much space.

In the theory part, 4 resource distribution metrics are introduced. These metrics are compared by comparing the solutions of the case study problems whose solutions are given below. The optimization process evaluates the objective functions of the resource distribution metrics and the obtained resource distribution diagram which provides the minimum objective function is presented as the optimum solution. In this study four resource distribution metrics are compared so that each case study problem is evaluated by four different objective functions whose formulations are given in Eq. 1 to 4 .

\subsection{First case study problem}

First case study problem is obtained from Geng et al. [16]. The project has nine activities in which five of them are uncritical. Project duration is twenty three days and the search domain is 272. Activity on arrow diagram of the first case study problem is given in Fig. 1. Durations and resource demand of the activities are shown in the diagram.

Text over the arrows represents the activity name. The first number represents the activity duration and the following numbers represent resource demand of the corresponding activity. The obtained optimum resource distribution diagrams 
by the tested resource distribution metrics are shown in Fig. 2.

In Fig. 2, blue bars represent the resultant resource distribution when all of the activities start at early start time. There are five bars for each day. The remaining four bars represent the optimized resource distribution with minimum moment $(\mathrm{MM})$, absolute difference (AD), minimum deviation (MD) and square of difference (SD) metrics respectively. The maximum daily resource demand cannot be decreased by any of the metrics.

When fluctuations of the resource distribution diagram are examined, it is seen that MM and MD metrics give the same resource distribution for the first 7 days. On the other hand, AD and SD metrics successfully level the idle workers between the $4^{\text {th }}$ and $7^{\text {th }}$ days. Moreover, these metrics provide stable increase and decrease throughout the construction period. The first problem is successfully leveled by $\mathrm{AD}$ and SD metrics while MM and MD metrics present poor performance.

\subsection{Second case study problem}

Second case study problem is obtained from Hinze [17]. Activity on arrow diagram is given in Fig. 3. This case study problem allocates four different resource types which are represented as R1, R2, R3, and $\mathrm{R} 4$ for the resource types $1,2,3$, and 4 respectively. Labels over the arrows gives information about the activities. First letter represents the activity label. The first number represents the activity durations and the consecutive 4 numbers represent the resource demand of the activity for the resource types R1, R2, R3, and R4 respectively. The project has fifteen activities in which six of them are uncritical. Project duration is seventeen days and the search domain is 600. In other words, by delaying the uncritical activities 600 feasible schedules can be obtained. The case study problem is solved by using the four resource distribution metric and the results are given in Fig. 4.

Initial peak resource demand for R1 is 12 . This demand is reduced to 9 by $\mathrm{MM}$ and 7 by $\mathrm{AD}, \mathrm{MD}$, and SD metrics respectively. Excessive resource demand at the second, eleventh and twelfth days are successfully leveled by all of the metrics. Consequently, MM provided the worst result for the R1.

Resource distribution diagram for $\mathrm{R} 2$ is given in Fig. 5. Initial peak resource demand for R2 is 17 . The peak resource demand is reduced to 12 by all of the four metrics. When the fluctuations of the daily resource distribution are examined it is seen that there are slight differences between the resource histograms. However, MM metric is slightly better than the others. MM metric reduces the fluctuations between the $12^{\text {th }}$ and $17^{\text {th }}$ days more efficiently.

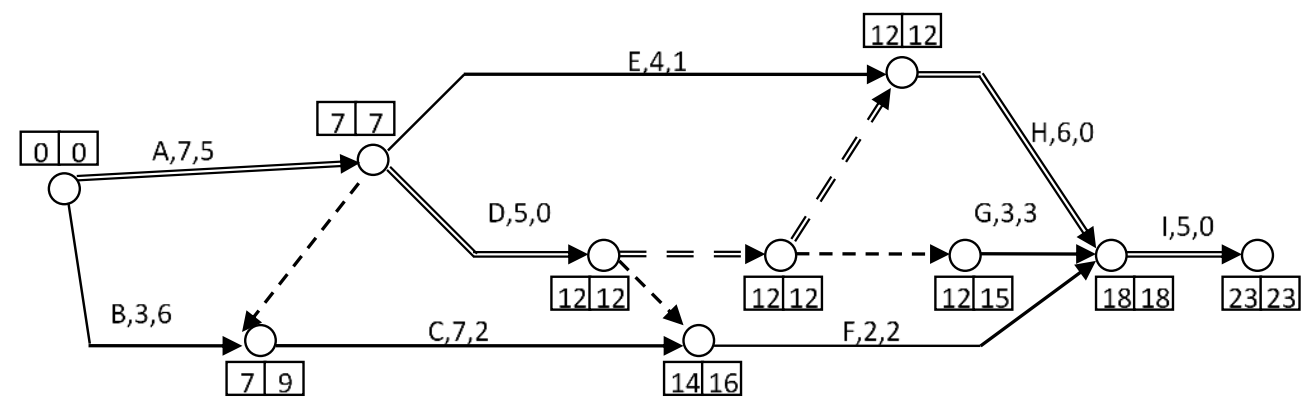

Fig. 1. Activity on arrow diagram of the first case study problem 


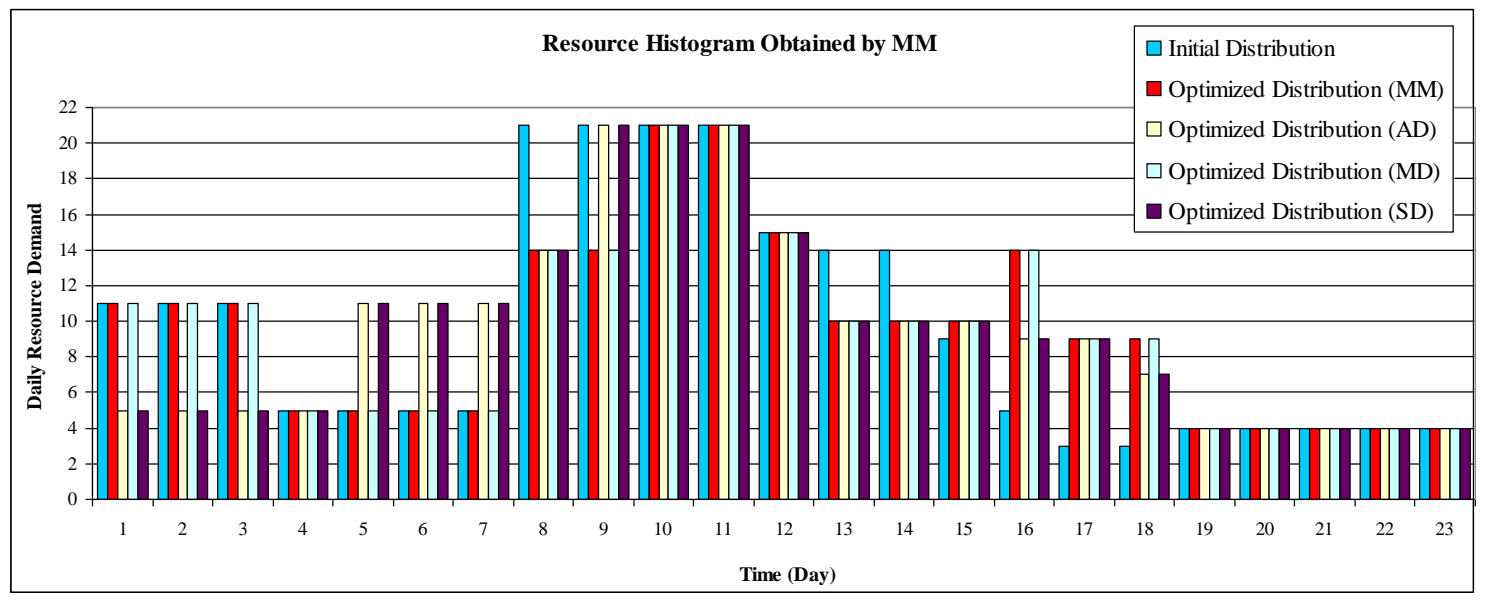

Fig. 2. Resource distribution of the first case study problem

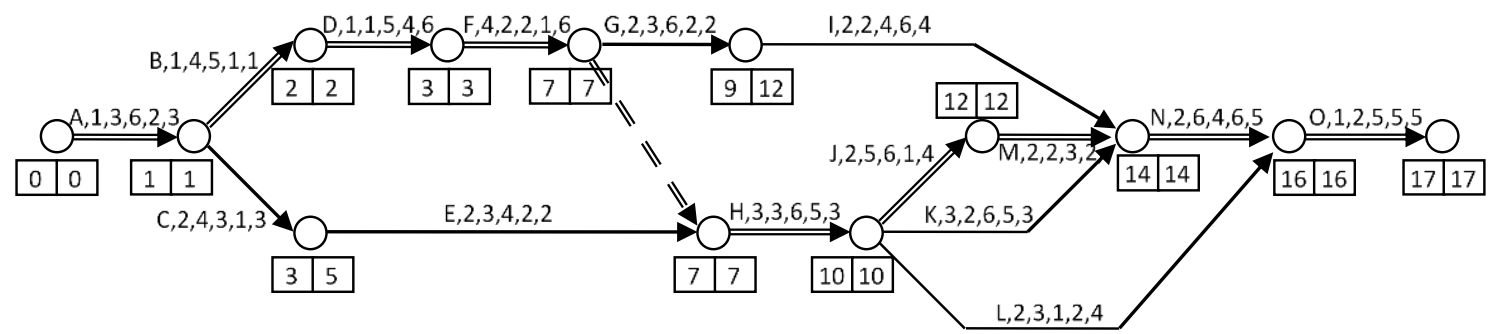

Fig. 3. Activity on arrow diagram of the second case study problem

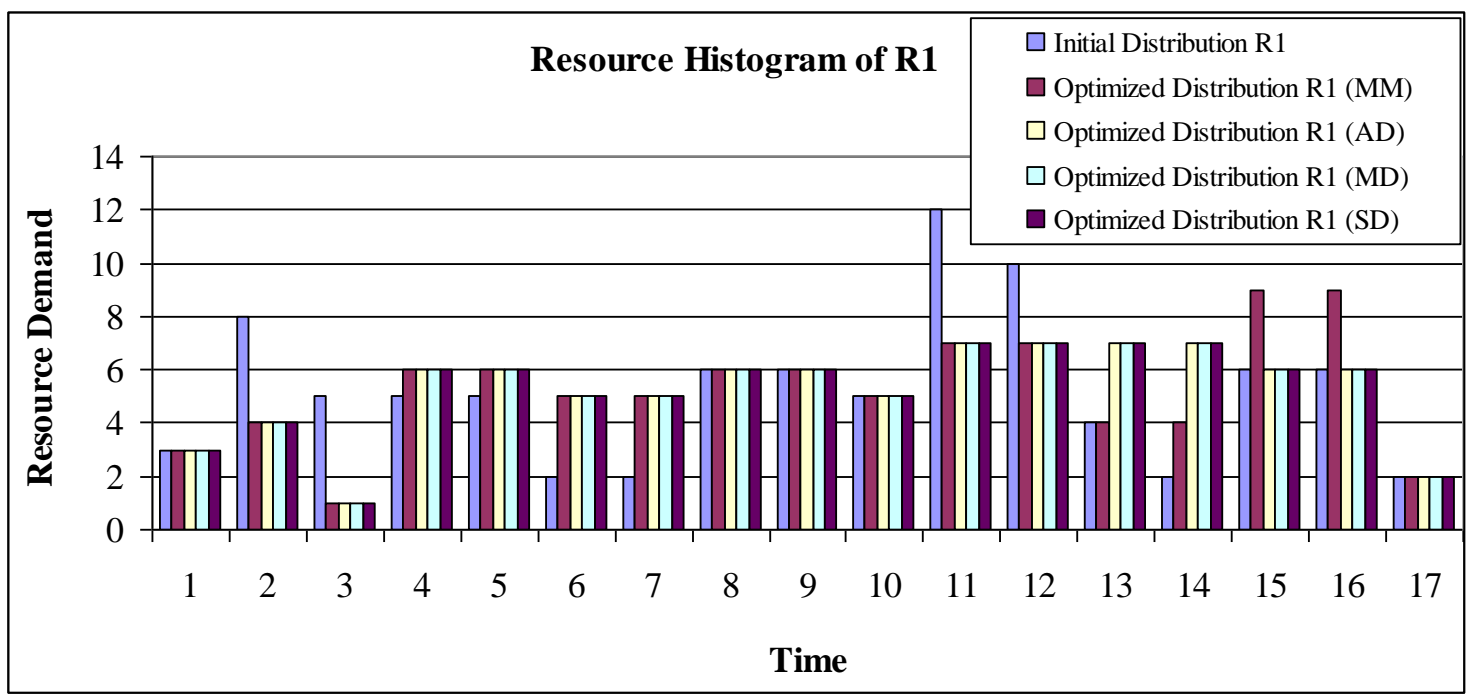

Fig. 4. Resource distribution diagram of R1 of the second case study problem 


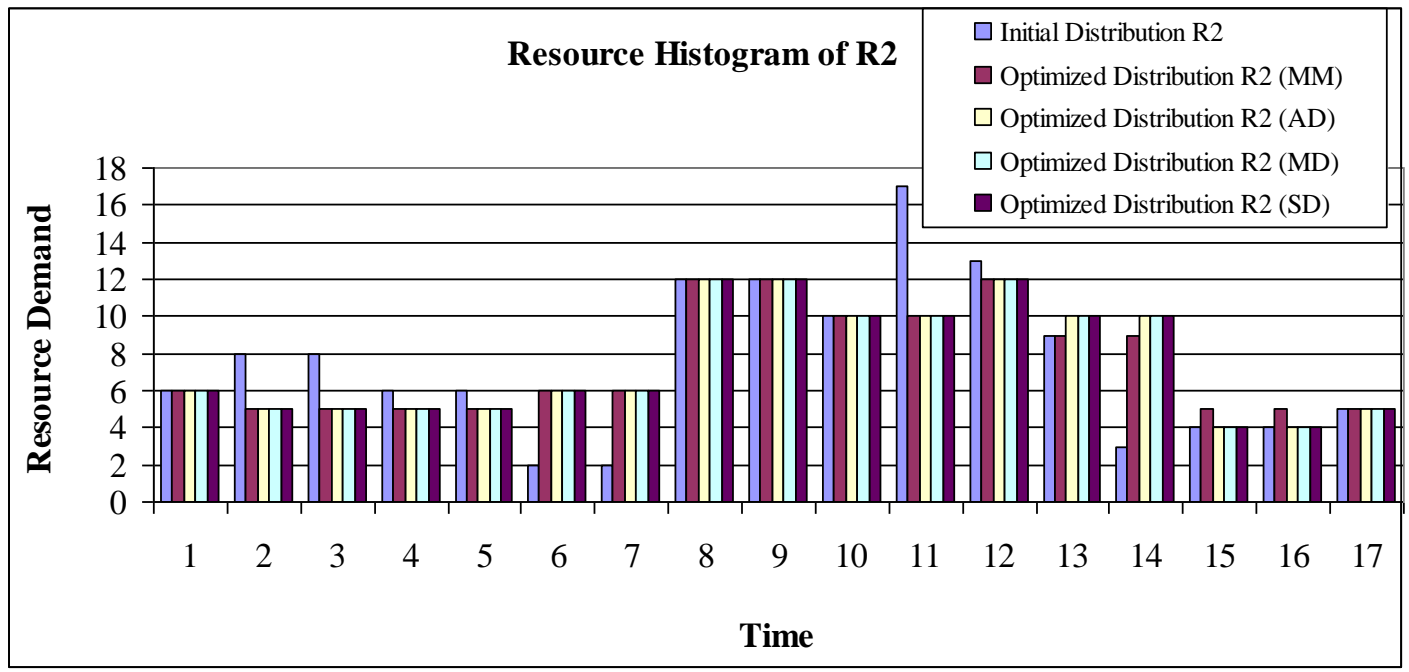

Fig. 5. Resource distribution diagram of $\mathrm{R} 2$ of the second case study problem

Fig. 6 represents the resource distribution histograms of R3. Initial peak resource demand of $\mathrm{R} 3$ is 14 . The peak demand is reduced to 11 by all of the metrics. The resource histogram is identical for all of the metrics between the first and twelfth days. On the other hand, between thirteenth and sixteenth days MM metric provides better resource distribution.

Resource distribution diagram of R4 is given in Fig. 7. Initial peak resource demand of R4 is 15 . The peak demand is reduced to 9 by all of the metrics. The resource distribution between the first and twelfth days is identical for all of the metrics. However, MM metric provides significantly a better resource distribution for the construction period between twelfth and sixteenth days. Number of idle resources and required hire-and-release is reduced by MM metric.

MM metric cannot successfully reduce the peak resource demand. However, this metric provided better performance when the reduction of fluctuations through the construction is considered.

\subsection{Third case study problem}

Third case study problem is obtained from Akpan [18]. Activity on arrow diagram of the project is given in Fig. 8. This project has two resource types. The project has eleven activities in which five of them are critical. Project duration is fifteen days and the search domain is 5684 . The case study problem is solved by using the four resource distribution metrics and the results are given in Figs. 9-10.

Resource distribution diagram of $\mathrm{R} 1$ is given in Fig. 9. Peak demand of R1 is 14 for the initial schedule. The peak demand cannot be reduced by MD metric. On the other hand, the peak demand for $\mathrm{R} 1$ is reduced to 9 by $\mathrm{MM}, \mathrm{AD}$, and SD metrics. MM metric provided a smooth resource distribution but this metric has the highest anomaly at the twelfth day of the construction. AD metric provides a better resource distribution than MM when only the fluctuations are considered. The impact like peak in the resource diagram is smoothed by AD. MD metric cannot smooth the histogram efficiently when its high peak resource demand is concerned. SD metric smoothes the resource distribution diagram the most efficiently for R1 among the examined metrics.

Fig. 10 represents the resource distribution diagram of R2. Peak demand of R2 is 8 for the initial schedule. The peak demand cannot be reduced by SD metric. This is because of the good performance at the leveling of resource type R1. It is seen that leveling of R1 negatively affected leveling of R2 for the SD metric. The peak demand is reduced to 6 by $\mathrm{MM}, \mathrm{AD}$, and $\mathrm{MD}$ metrics. The resource distributions for the aforementioned metrics are identical. 


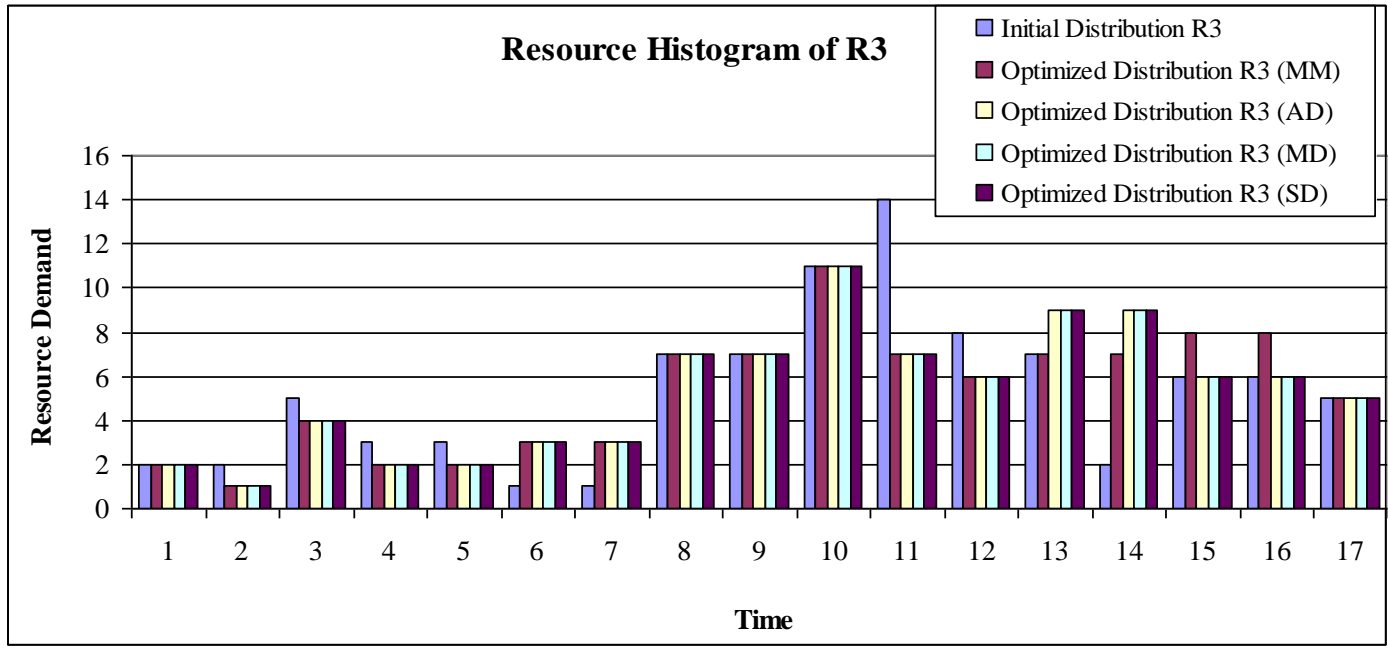

Fig. 6. Resource distribution diagram of R3 of the second case study problem

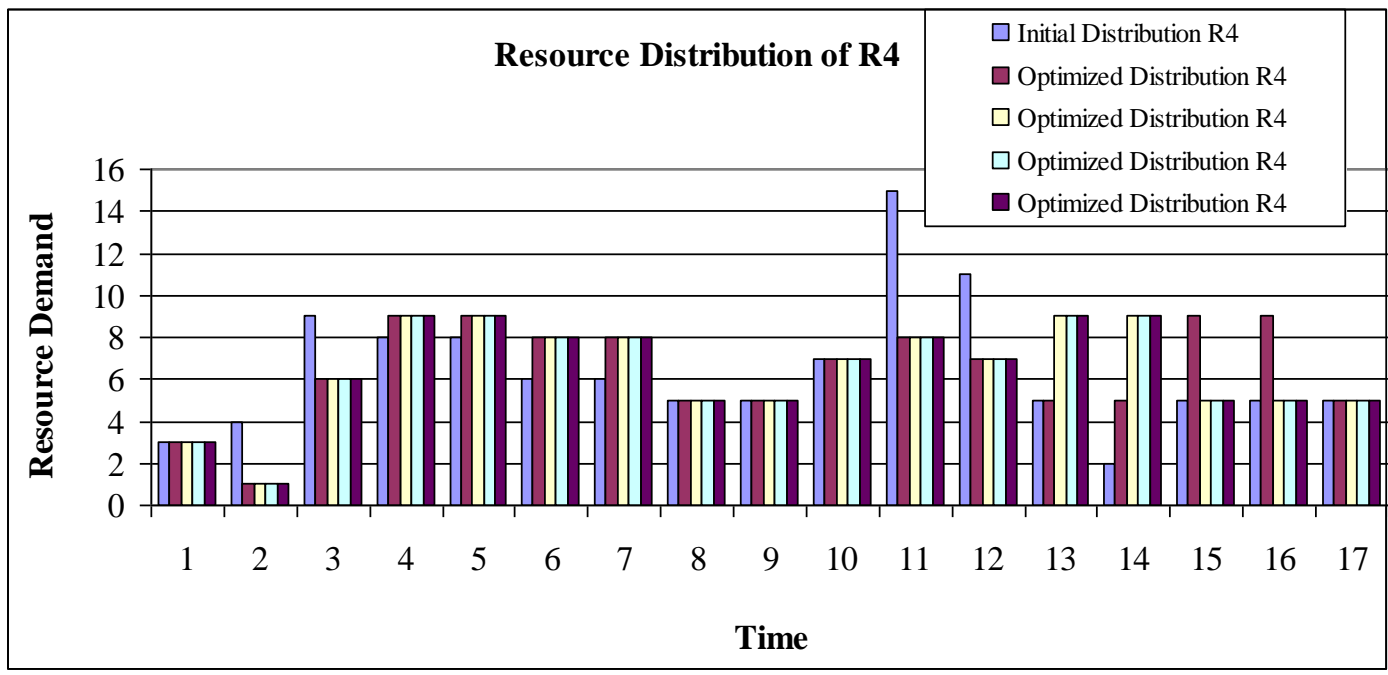

Fig. 7. Resource distribution diagram of R4 of the second case study problem

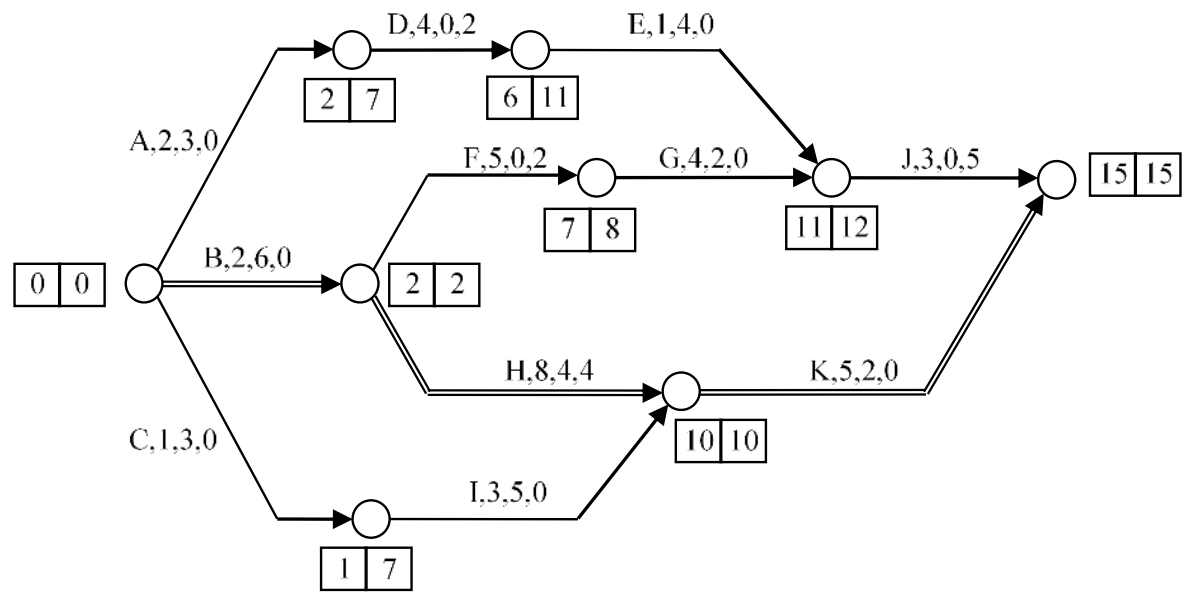

Fig. 8. Activity on arrow diagram of the third case study problem 


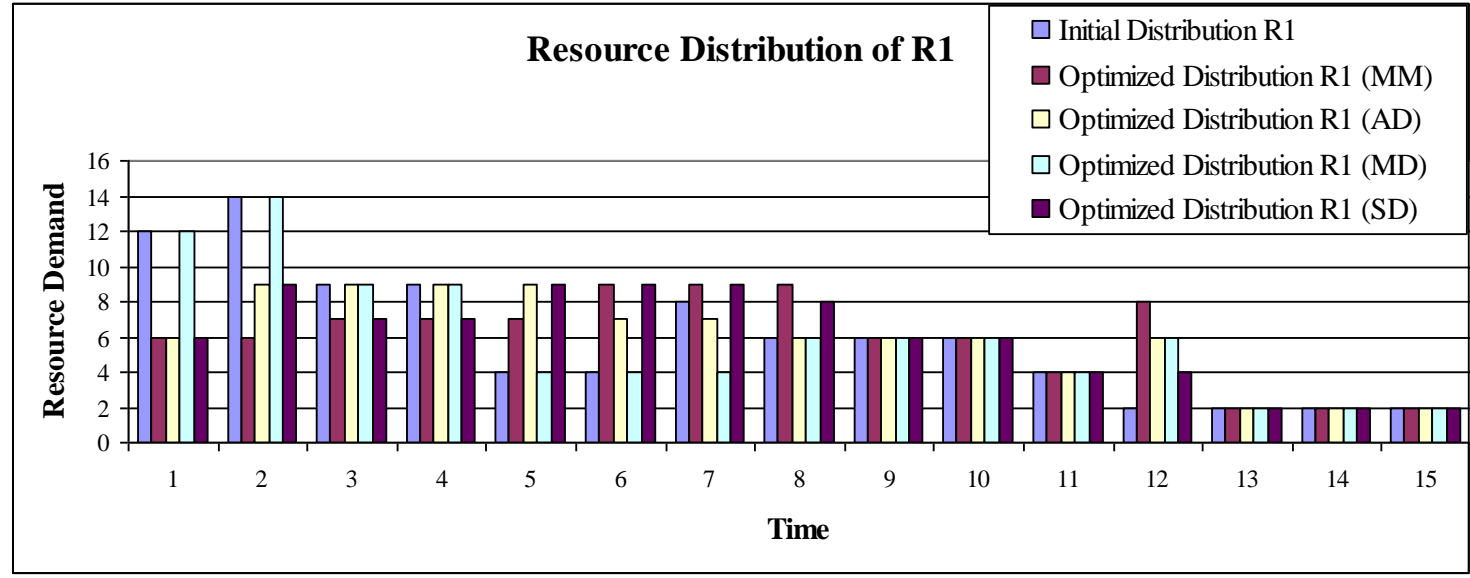

Fig. 9. Resource distribution diagram of R1 of the third case study problem

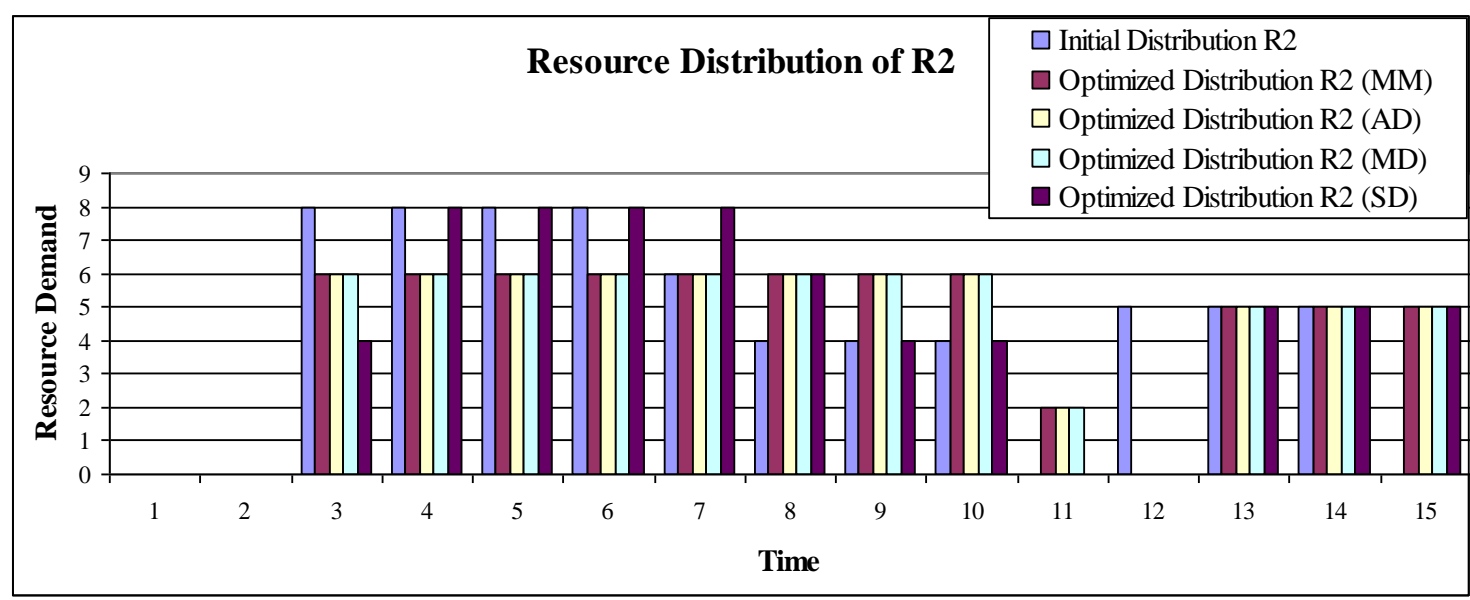

Fig. 10. Resource distribution diagram of $R 2$ of the third case study problem

\subsection{Fourth case study problem}

Fourth case study problem is obtained from Son and Skibniewski [19]. Activity on arrow diagram is given in Fig. 11. The project has eleven activities in which five of them are critical. Project duration is twenty three days and the search domain is 7134 . The case study problem is solved by using the four resource distribution metrics and the results are given in Fig. 12.

The peak resource demand of the initial schedule is 11 . The peak resource demand is reduced to 9 by MM, MD, and SD metrics, besides the peak resource demand is reduced to 8 by $\mathrm{AD}$ metric. The fluctuations of the resource histogram are almost the same for all metrics. The performance of $\mathrm{AD}$ is better than the others for the fourth case study.

\section{Conclusion}

In this study, performances of four resource distribution metrics; minimum moment, absolute difference, minimum deviation, and sum of squares are compared. Four case study problems obtained from the literature are used for the comparison. The examined problems are relatively small projects when compared with real construction projects. However, the case study problems are larger than the recent study conducted by Bettemir and Erzurum [15]. 


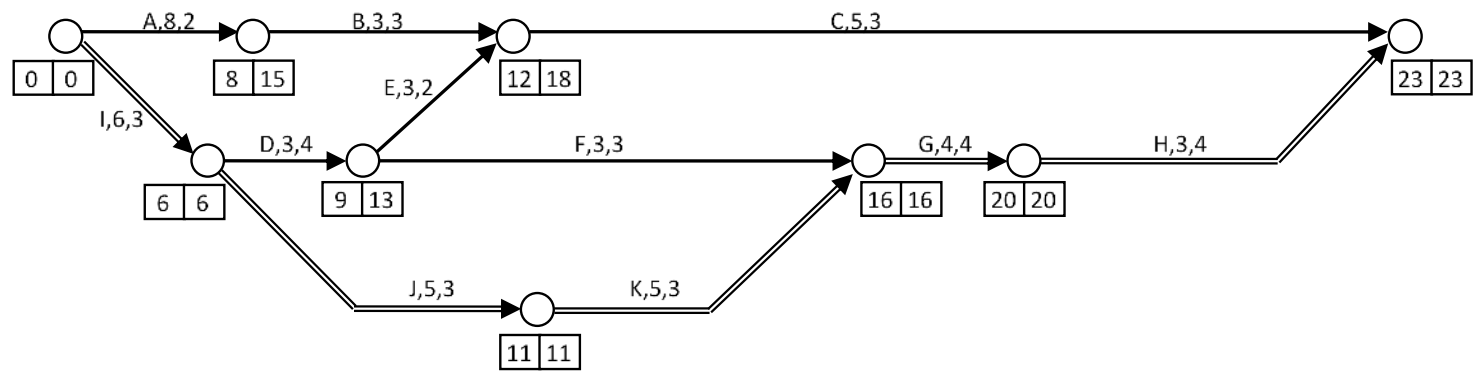

Fig. 11. Activity on arrow diagram of the fourth case study problem

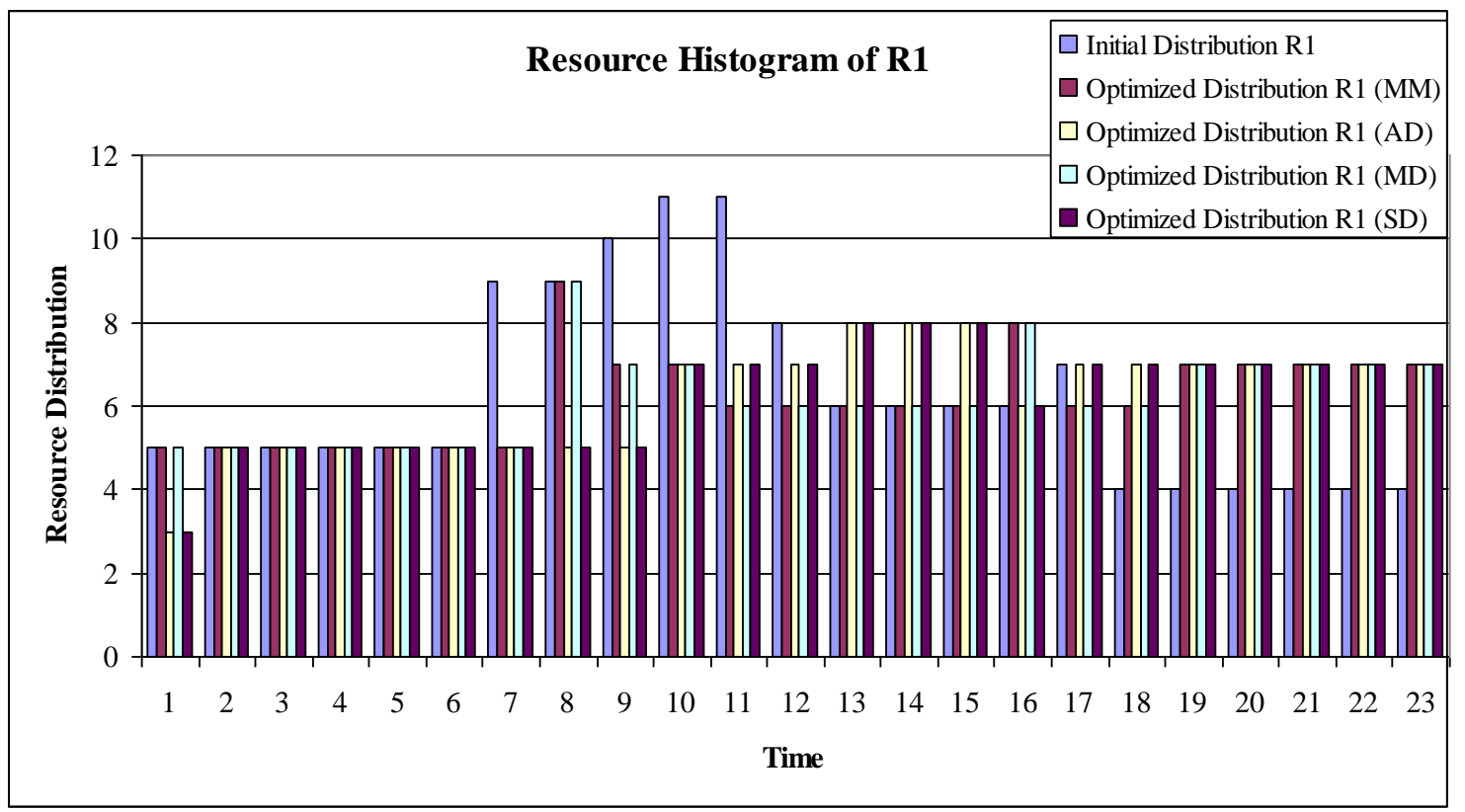

Fig. 12. Resource distribution diagram of the fourth case study problem

The comparison is made by taking the reduction in the initial peak resource demand and the lessening of the rises and falls throughout the daily resource demand into account. The analysis results reveal that minimum deviation and sum of squares metrics cannot improve the initial resource distribution histogram obtained by commencing the activities at early start times. Therefore, endeavor of optimization do not provide the expected benefit when the aforementioned resource leveling algorithms are implemented.

Absolute Difference and Minimum Moment metrics provided better performance. These metrics reduced the peak resource demand more effectively. In addition to this, fluctuations of the resource distribution during the construction period are decreased more efficiently. This leads to less idle resources and fewer release-and-rehire. As a result, construction costs can be reduced. Four case study problems are not adequate to make a conclusion but $\mathrm{AD}$ provided smaller peak resource demands than MM. Therefore AD metric can be considered as the best resource distribution metric among the compared ones.

The output of this study can be beneficial for construction managers for the selection of resource distribution metric, since this study show that the selection of proper metric is vital to reduce the peak 
resource demand. The comparison is made by using four case study problems. The sample size is not adequate for obtaining a certain idea. This study can be repeated by collecting more sample projects from the literature as future study. In addition to this, the search domain of the tested problems was not large and resource distribution histograms did not diversify enough. Hypothetic project can be factorized whose search domains are larger than the tested sample problems.

\section{References}

[1] Rieck J, Zimmermann J, Gather T (2012). Mixedinteger linear programming for resource leveling problems. European Journal of Operational Research, 221(1), 27-37.

[2] Neumann K, Schwindt C, Zimmermann J (2012). Project scheduling with time windows and scarce resources: temporal and resource-constrained project scheduling with regular and nonregular objective functions. Springer Science \& Business Media.

[3] Karaa FA, Nasr AY (1986). Resource management in construction. J. Constr. Eng. Manage., 112(3), 346-357.

[4] Easa SM (1989). Resource leveling in construction by optimization. J. Constr. Eng. Manage., 115(2), 302-316.

[5] Demeulemeester E, Herroelen W (1992). A Branch-and-Bound Procedure for the Multiple Resource-Constrained Project Scheduling Problem. Manage. Sci., 38(12), 1803-1818.

[6] Bandelloni M, Tucci M, Rinaldi R (1994). Optimal resource leveling using non-serial dynamic programming. Eur. J. Oper. Res., 78(2), 162-177.

[7] Neumann K, Zimmermann J (1999). Resource Levelling for Projects with Schedule-Dependent Time Windows. Eur. J. Oper. Res., 117(3), 591605.

[8] Mutlu MÇ (2010). A branch and bound algorithm for resource leveling problem, MSc Thesis, METU.

[9] Erzurum T. Optimum Or Near-Optimum Solution Of Resource Leveling Problems With Spreadsheet Application, Master of Science Thesis, İnönü University, Malatya, Turkey, 2019.

[10] Burgess AR, Killebrew JB (1962). Variation in activity level on a cyclical arrow diagram. J. Indust. Eng., 13(2), 76-83.

[11] Ahuja HN. Construction performance control by networks, Wiley, NY, 1976.
[12] El-Rayes K, Jun DH (2009). Optimizing resource leveling in construction projects. J. Constr. Eng. Manage., 135(11), 1172-1180.

[13] Erzurum T, Bettemir ÖH. Determination of Search Domain Of Resource Leveling Problem, International 7th Construction Management Congress, Samsun, 2017.

[14] Erzurum T, Bettemir ÖH. Optimum or NearOptimum Resolution of Resource Leveling Problems with Spreadsheet Application, 5th International Project and Construction Management Conference - IPCMC2018, Cyprus International University, Faculty of Engineering, Civil Engineering Department, North Cyprus, 2018.

[15] Bettemir ÖH, Erzurum T. Comparison of Resource Distribution Metrics On Small Projects. International Civil Engineering and Architecture Conference (ICEARC'19), 17-20 April (2019), Trabzon, Türkiye.

[16] Geng JQ, Weng LP, Liu SH (2011). An improved ant colony optimization algorithm for nonlinear resource-leveling problems. Computers \& Mathematics with Applications, 61(8), 2300-2305.

[17] Hinze JW. Construction Planning and Scheduling, 3rd Edition, Pearson Prentice Hall, Upper Saddle River, NJ, 2006.

[18] Akpan EOP (2000). Resource smoothing: a cost minimization approach. Production Planning \& Control, 11(8), 775 - 780.

[19] Son J, Skibniewski MJ (1999). Multiheuristic approach for resource leveling problem in construction engineering: Hybrid approach. Journal of Construction Engineering and Management, 125(1), 23-31. 